\title{
Thermal Phase Lag Heterodyne Infrared Imaging for Current Tracking IN RADIO FREQUENCY INTEGRATED CIRCUITS
}

X. Perpiñà ${ }^{1}$, J. León ${ }^{1}$, J. Altet ${ }^{2}$, M. Vellvehi ${ }^{1}, F$. Reverter $^{2}$, E. Barajas $^{2}$, X. Jordà ${ }^{1}$.

${ }^{1}$ Centre Nacional de Microelectrònica IMB-CNM (CSIC), Campus UAB, 08193 Bellaterra (Barcelona), Spain

${ }^{2}$ Departament d'Enginyeria Electrònica, Universitat Politècnica de Catalunya, Barcelona 08034, Spain.

\section{Abstract}

With thermal phase lag measurements, current paths are tracked in a Class A Radio Frequency (RF) power amplifier at $2 \mathrm{GHz}$. The amplifier is heterodynally driven at 440 $\mathrm{MHz}$ and $2 \mathrm{GHz}$, and its resulting thermal field respectively inspected at 1013 and 113 $\mathrm{Hz}$ with an infrared lock-in thermography system. The phase lag maps evidence with a higher sensitivity than thermal amplitude measurements, an input-output loop due to a substrate capacitive coupling. This limits the amplifier's performance, raising the power consumption in certain components. Other information relative to local power consumption and amplifier operation is also inferred. This approach allows the local non-invasive testing of integrated systems regardless of their operating frequency. 
Layout studies in Radio Frequency (RF) Integrated Circuits (ICs) are crucial to understanding their performance limits ${ }^{1}$ and ageing effects. ${ }^{1,2}$ Traditionally, the IC local behavior has been inferred from high level electrical figures of merit; which requires expensive equipment, complex tests, and access to internal nodes with frequency adapted pads. $^{3,4,5}$ Unfortunately, this approach cannot always determine intradie parasitic couplings responsible for high frequency undesired current paths, whose detection and prevention is a cornerstone in RF ICs analog design (mainly in power amplifiers). ${ }^{1,6,7}$ Such paths can limit the IC frequency response and power delivered to the load, giving rise to overloading conditions and eventually, the IC failure. ${ }^{8}$ To identify them, an innovative and efficient solution is its local tracking through the IC under operation. In this regard, using off-chip thermal imaging systems (e.g., Infrared Lock-In Thermography ${ }^{9}$-IR-LIT-, thermoreflectance ${ }^{10}$, or MOSFET hot-carriers' luminescence ${ }^{11}$ ) is very promising. ${ }^{12,13,14,15,16,17}$ They noninvasively sense the heat generated while the IC is under operation. Besides, the Joule effect allows converting electrical information from a high frequency $f$ into a thermal field at a lower frequency $\Delta f$ (heterodyne detection) within the imaging system bandwidth. ${ }^{13,17,18,19}$ Currently, such techniques are only employed for failure analysis and debugging, ${ }^{13,17,18,20,21,22,23,24}$ whereas maximum frequencies of $30 \mathrm{MHz}$ in test structures ${ }^{19}$ and around $14 \mathrm{MHz}$ in IC powering systems ${ }^{17}$ have been reported. They only monitor the thermal amplitude of hot spots, where thermal phase lag only assists to locate them through the following criterion: a maximum in amplitude and a minimum in phase lag should be detected at the same place. ${ }^{17,19}$ As a new application scenario, this work proposes tracking the current paths in more complex systems (RF ICs) operating within GHz range by thermal phase lag measurements. In comparison with amplitude monitoring, phase lag is surface emissivity independent ${ }^{9}$ and more sensitive to heat sources detection (thermal activity) 
when synchronized with a driving electrical signal.

To illustrate this, let us consider an IC with $N$ devices located on top of a semiconductor die at several positions $\vec{r}_{i}$. The IC input is driven with a voltage $V_{\text {gen }}(t)$, which allows heterodyne excitation, i.e.: $V_{\text {gen }}(t)=V_{\text {gen, } 0}\left(\cos \left(2 \pi f_{1} t\right)+\cos \left(2 \pi f_{2} t\right)\right)$ such that $\Delta f=f_{1}-f_{2} \ll f_{1}, f_{2}$ and $f=f_{1} \approx f_{2}$. These devices may behave as thermally interacting heat sources and leading to a total thermal field $\Delta T(\vec{r}, t)$ at any given location on the die surface $\vec{r}$. The $i$-th heat source withstands a voltage drop $V_{i}(f)$ and current $I_{i}(f)$, and dissipates, due to Joule effect, a real power spectral component at $\Delta f P_{i}(t):{ }^{19}$

$$
P_{i}(t)=\left|V_{i}(f)\right|\left|I_{i}(f)\right|\left|\cos \left(\eta_{i}(f)\right)\right| \cos \left(2 \pi \Delta f t+\vartheta_{i}+\chi\right)
$$

where $\eta_{i}(f)$ is the electrical phase lag between $V_{i}(f)$ and $I_{i}(f), \vartheta_{i}$ corresponds to the phase lag of the heat generated (or power dissipated) between each heat source (or device), and $\chi$ provides the phase lag respect to $V_{\text {gen }}(t)$. Thus, the $i$-th heat source generates a thermal field spectral component at $\Delta f\left[\Delta T_{i}\left(\left|\vec{r}-\vec{r}_{i}\right|, \Delta f\right)\right]$ with an amplitude $\left|\Delta T_{i}\left(\left|\vec{r}-\vec{r}_{i}\right|, \Delta f\right)\right|$ and phase lag $\theta_{i}\left(\left|\vec{r}-\vec{r}_{i}\right|, \Delta f\right)+\vartheta_{i}$. When all heat sources thermally interact, the amplitude and phase lag of the spectral component at $\Delta f$ of the thermal field resulting from their thermal interaction $\left[|\Delta T(\vec{r}, \Delta f)|\right.$ and $\phi_{\tau}(\vec{r}, \Delta f)$, respectively] writes as:

$$
\begin{aligned}
|\Delta T(\vec{r}, \Delta f)|= & {\left[\sum_{i=1}^{N}\left|\Delta T_{i}\left(\left|\vec{r}-\vec{r}_{i}\right|, \Delta f\right)\right|^{2}\right.} \\
& +2 \sum_{i \neq l}^{N}\left|\Delta T_{i}\left(\left|\vec{r}-\vec{r}_{i}\right|, \Delta f\right)\right|\left|\Delta T_{l}\left(\left|\vec{r}-\vec{r}_{l}\right|, \Delta f\right)\right| \cos \left[\theta_{i}\left(\left|\vec{r}-\vec{r}_{i}\right|, \Delta f\right)+\vartheta_{i}\right. \\
& \left.\left.-\left(\theta_{l}\left(\left|\vec{r}-\vec{r}_{l}\right|, \Delta f\right)+\vartheta_{l}\right)\right]\right]^{1 / 2}
\end{aligned}
$$




$$
\phi_{\tau}(\vec{r}, \Delta f)=\tan ^{-1}\left[\frac{\sum_{i=1}^{N}\left|\Delta T_{i}\left(\left|\vec{r}-\vec{r}_{i}\right|, \Delta f\right)\right| \sin \left(\theta_{i}\left(\left|\vec{r}-\vec{r}_{i}\right|, \Delta f\right)+\vartheta_{i}\right)}{\sum_{i=1}^{N}\left|\Delta T_{i}\left(\left|\vec{r}-\vec{r}_{i}\right|, \Delta f\right)\right| \cos \left(\theta_{i}\left(\left|\vec{r}-\vec{r}_{i}\right|, \Delta f\right)+\vartheta_{i}\right)}\right]
$$

where equations (1)-(3) link $|\Delta T(\vec{r}, \Delta f)|$ and $\phi_{\tau}(\vec{r}, \Delta f)$ to the high frequency electrical signals flowing through each device. For reliable lock-in measurements, both $\phi_{\tau}(\vec{r}, \Delta f)$ and $\chi$ are mandatory to be stable or noiseless, even when low or null $|\Delta T(\vec{r}, \Delta f)|$ is detected. When given, this situation points out a certain thermal activity or heat generation in correlation with the reference signal $V_{\text {gen }}(t)$; and $\phi_{\tau}(\vec{r}, \Delta f)$ measurements can indicate current paths (whenever thermal activity is detected) and power flows (i.e., absorbed -positive- or delivered -negative- power from the biasing source $)^{25}$. In Eq. (1), when $\vartheta_{i}$ is only induced by $\eta_{i}(f), \vartheta_{i}=\cos ^{-1}\left[\operatorname{sgn}\left(\cos \left(\eta_{i}(f)\right)\right]\right.$ is fulfilled, where $\operatorname{sgn}\left(\cos \left(\eta_{i}(f)\right)\right.$ is the sign function of $\cos \left(\eta_{i}(f)\right.$. Therefore, $\vartheta_{i}$ can only be 0 or $\pi$ rad. This extremely affects the spatial response of $\phi_{\tau}(\vec{r}, \Delta f)$, as $\theta_{i}\left(\left|\vec{r}-\vec{r}_{i}\right|, \Delta f\right)$ has a weaker dependence on $\left|\vec{r}-\vec{r}_{i}\right|$. Consequently, $\phi_{\tau}(\vec{r}, \Delta f)$ spatial profiles present an abrupt transition with a large signal variation, which enhances the measurement dynamic range, as in the thermal gradient monitoring, ${ }^{14}$ and provides a higher immunity to noise than those of $|\Delta T(\vec{r}, \Delta f)|{ }^{26}$ As a proof of concept, this work proposes to locally study by IR-LIT the current paths of a Class A RF power amplifier at $f=2 \mathrm{GHz}$ to determine an input-output loop due to a substrate capacitive coupling. IR-LIT has been selected because of its commercial availability, minimum requirements in sample preparation, thermal sensitivity, ${ }^{14}$ and capability of carrying out heterodyne detection. ${ }^{19}$

Figure 1 depicts the schematic (a) and the layout (b) of the power amplifier under study: 2 transistors in a cascode topology, with an isolated DC output and an L-C band input (implemented in Figure 1 with $L_{\text {in }}$ and $C_{\text {in }}$ ) frequency tuning. The transistors role in the amplifier is the following. $M_{1}$ is a DC self-biased (through resistor $R_{1}$ ) transistor in common source, which converts the applied input gate voltage into an output drain 
current to reach the amplifier power gain required in the load (e.g., antenna). By contrast, $M_{2}$, in common gate, isolates the output node and $M_{1}{ }^{27}$ This amplifier has been fabricated in $0.35 \mu \mathrm{m} \mathrm{2P4M} \mathrm{(2} \mathrm{levels} \mathrm{of} \mathrm{polysilicon,} 4$ levels of metal) Complementary Metal-Oxide-Semiconductor (CMOS) technology onto a $725 \mu \mathrm{m}$ thick substrate and tuned at a central frequency $f_{\mathrm{c}, \mathrm{G}}=440 \mathrm{MHz}$. For the experiments, the amplifier input has been heterodynally driven with an Agilent E4438C ESG Vector Signal Generator (total input power $\left|P_{\text {in,het }}\right|$ of $0.5 \mathrm{~mW}$ ), and a FLIR SC5500 IR camera has been employed for thermal acquisitions. A frame rate of $376 \mathrm{~Hz}$ and an integration time of $1200 \mu \mathrm{s}$ have been considered (i.e.: $2.5 \times 10^{4}$ images processed at $\Delta f$ ). To improve the signal to noise ratio (SNR) during IR measurements, the die, attached to a specifically-designed RF board, has been placed on a micropositioning stage thermoregulated with a Peltier system and its operating temperature $T_{\mathrm{i}}$ has been set for $60^{\circ} \mathrm{C}$. As a result, the maps of $|\Delta T(\vec{r}, \Delta f)|$ and $\phi_{\tau}(\vec{r}, \Delta f)$ (referred to as $|\Delta T|$ and $\phi_{\tau}$ ) were derived. The so-called $|\Delta T|$ apparent maps $\left(|\Delta T|_{\text {app }}\right)$ have been also used, as they allow straightforward analyzing the electrical behavior when IC locations or areas with the same surface emissivity are considered.

The capacitive input-output loop has been studied by comparing $|\Delta T|_{\text {app }}$ at $f=f_{\mathrm{c}, \mathrm{G}}$ [Figure 2 (a)] with $|\Delta T|_{\text {app }}$ and $\phi_{\tau}$ at $f=2 \mathrm{GHz}$ [Figures 2 (b) and (c), respectively] setting the appropriate $\Delta f$ in each case. For $f=f_{\mathrm{c}, \mathrm{G}}, \Delta f=1013 \mathrm{~Hz}$ was selected to confine the thermal field around the heat sources, whereas for $f=2 \mathrm{GHz}, \Delta f=113 \mathrm{~Hz}$ was set to obtain during IR-LIT acquisitions, an optimum SNR ensuring the measurement independence from boundary conditions. ${ }^{14,19}$ Amplitude results are first used to identify which amplifier devices behave as hot spots at two different frequencies. As explained latter, phase measurements will assist in determining high frequency parasitic capacitive couplings which are not appearing at the central frequency. Fir this reason, $\phi_{\tau}$ at 
$f=440 \mathrm{MHz}$ is not provided. In Figure 2 (a), transistors $M_{1}$ and $M_{2}$, jointly with the capacitor $C_{\text {ext,2 }}$ (power delivered to the output), are the components with higher consumption during regular operation. This contrasts with Figure 2 (b) where $L_{\text {in }}$ and $M_{2}$ are the main heat sources, as also observed in $\phi_{\tau}$ map [Figure 2 (c)]. This indicates that at $f=2 \mathrm{GHz}, i_{\text {in }}$ is mainly diverted across a capacitive coupling path [Path A], while that expected without any input-output loop [Path B] is still thermally detectable. To better interpret such results, a high frequency small-signal equivalent circuit has been inferred in Figure 2 (d) for Paths A (in solid blue) and B (in dashed red). These qualitative description considers: the $L_{\text {in }}$ series resistance $\left(R_{L \mathrm{par}}\right)$, transconductance $g_{m, M_{m}}$ for each transistor (i.e., $m=1,2$ ), which relates the drain current $i_{D, m}$ with the gate to source voltage $v_{g s, m}$, and its parasitic capacitances (i.e., gate to drain, $C_{g d m}$, and gate to source, $\left.C_{g s m}\right)^{28}$. In Figures 2 (b) and (c), Path A is observed below $C_{\text {in }}$ and between the hottest areas corresponding to $L_{\text {in }}$ and $M_{2}$. Additionally, other local heat sources $\left(H S_{1}\right.$ and $\left.H S_{2}\right)$ appear. $H S_{1}$ corresponds to the interconnecting via between metal levels 3 and 4 in $L_{\text {in }}$, whereas $H S_{2}$ locates a hot spot along the connection track (metal level 3) between $L_{\text {in }}$ and $C_{\text {in }}$ only observable as a local minimum in $\phi_{\tau}$ map. These results differently reveal the origin of the $L_{\mathrm{in}}-M_{2}$ capacitive path when the substrate equivalent impedance [ $R_{\text {subs }}$ in series with $C_{\text {subs, }}$ Figure 2 (d)] decreases with $f$. In $|\Delta T|_{\text {app }}$ map, the coupling is mostly located between $H S_{2}$ and $M_{2}$ (below $C_{\text {in }}$ ) and between $H S_{1}$ and $M_{2}$, showing a noisy spatial acquisition. By contrast, a larger area in figure 2 (c) depicts the coupling in $\phi_{\tau}$ map with a much better spatial SNR. On the other hand, Path B is pointed out only in $\phi_{\tau}$ map [Figure 2 (c)], where $H S_{2}$ and $C_{\text {in }}$ thermal activities are detected (local minimum in $\phi_{\tau}$ reached). When $|\Delta T|_{\text {app }}$ is practically null in some IC components (e.g., $R_{2}, C_{\text {ext, }, 1}$, $R_{1}, C_{\text {in }}$ or $M_{1}$ ), additional information can be extracted from $\phi_{\tau}$ maps with a higher dynamic range, e.g., $R_{2}-C_{\text {ext, } 1}$ branch, $R_{1}, C_{\text {in }}$ and $M_{1}$ are thermally active. This 
evidences an extremely low current level (almost negligible) passing across them, as the power consumption in such components is practically null according to $|\Delta T|_{\text {app }}$ map. Consequently, at $f=2.0 \mathrm{GHz}, i_{\text {in }}$ mostly passes across $L_{\text {in }}$ and $M_{2}$, avoiding $M_{1}$. Accordingly, the following can be inferred: when $i_{\text {in }}$ starts to divert through Path A, $V_{g s 1}$, and consequently, $V_{d s 1}$ and $V_{s g 2}$, decrease, lowering the power dissipated by $M_{1}$ [Figures 2 (c) and (d)]. On the contrary, $M_{2}$ conducts both the currents from Paths A and $\mathrm{B}$, as it operates as a cascode (resistor-like element) extremely increasing its heat dissipation [Figures 2 (c) and (d)]. Simultaneously, the impedance in $L_{\text {in }}$ increases with frequency, which enlarges its voltage drop or $v_{\mathrm{L}, \text { par }}$ [Figures 2 (b), (c), and (d)], and consequently, its power dissipation.

Thanks to the higher sensitivity and larger dynamic range of $\phi_{\tau}$ measurements, deeper details can be deduced about the IC power flow and $M_{2}-L_{\text {in }}$ coupling. Figure 3 depicts a $\phi_{\tau}$ map superposed to the IC layout (a), reporting two $\phi_{\tau}$ profiles: the former along $M_{1}$ and $M_{2}\left[(\mathrm{~b})\right.$, referred to as $\left.\left.\phi_{\tau}\right|_{M_{2}}\right]$, and the latter between both transistors and $L_{\text {in }}\left[(\mathrm{c})\right.$, referred to as $\left.\left.\phi_{\tau}\right|_{L_{i n}}\right]$. Moreover, Figure 3 (c) presents a data averaging performed for $x<360 \mu \mathrm{m}$ (black solid lines) to illustrate the sensitivity of this approach for a spatial noisy acquisition. With regards to the power flow, $\left.\phi_{\tau}\right|_{M_{2}}$ points out without any spatial filtering, an abrupt transition coincident with the $M_{1}$ placement, and its minimum is aligned with $M_{2}$ location, i.e., the main heat source along $\left.\phi_{\tau}\right|_{M_{2}}$ [Figure 3 (b)]. According to the presented theory, this steep variation is consequence of $\vartheta_{M_{1}}-\vartheta_{l}=\pi \operatorname{rad}(l$ refers to any other IC component), and only occurs when $\eta_{M_{1}}=\pi \mathrm{rad}$ and $\vartheta_{M_{1}} \gg \vartheta_{l}, \theta_{M_{1}}, \theta_{l}$. Therefore, $M_{1}$ is still delivering electrical power from the biasing sources to the rest of IC components, though the heat generation is very low as derived from $|\Delta T|_{\text {app }}$ map [Figure 2 (b)]. This is confirmed with the behavior of other IC components thermally active in Figure 3 (a), which are almost at the same 
$\phi_{\tau}$ than $M_{2}$. As for $L_{\mathrm{in}}-M_{2}$ coupling, $\left.\phi_{\tau}\right|_{L_{\text {in }}}$ presents a local relative minimum between $H S_{1}$ and $H S_{2}$ [Figure 3 (c)], which indicates the parasitic path position in the IC [necessary condition according to Eq. (3)]. The local modulation in $\left.\phi_{\tau}\right|_{L_{\text {in }}}$ minimum observed in Figure 3 (c) allows correlating not only the $H S_{1}$ and $H S_{2}$ locations with layout interconnecting elements, but also the track vias to $C_{\text {in }}$ (via, $C_{\text {in }}$ ) and $L_{\text {in }}$ output (via) in $H S_{2}$. According to this figure, $H S_{1}, H S_{2}$, and track vias in $H S_{2}$ are the leakage current spots responsible for the substrate capacitive coupling.

$\phi_{\tau}$ also allow studying the thermal range between heat sources to consider whether they interact. $\left.\phi_{\tau}\right|_{M_{2}}$ and $\left.\phi_{\tau}\right|_{L_{i n}}$ show for $x \geq 360 \mu \mathrm{m}$, a linear trend on lateral position $x$, whose slope follows the equation: ${ }^{19}$

$$
\alpha=1 / L_{\mathrm{D}}=\sqrt{\pi \Delta f / D_{\alpha}} .
$$

$\alpha$ corresponds to the inverse of the thermal characteristic length $L_{\mathrm{D}}$ defining the heat confinement radius around a non-interacting heat source, and $D_{\alpha}$ is the Silicon thermal diffusivity $\left(85.49 \pm 2.41 \mathrm{~mm}^{2} \mathrm{~s}^{-1}\right.$, used in this work). ${ }^{29}$ According to this $D_{\alpha}$ value and Eq. (4), $\alpha$ is $(2.04+0.06) \times 10^{-3} \mu \mathrm{m}^{-1}$, which perfectly agrees with that experimentally inferred from Figures 3 (b) and (c) ( $\alpha_{1}$ and $\alpha_{2}$, respectively). This reveals two important conclusions on spatial noise and thermal interaction range. Firstly, even in the noisiest $\phi_{\tau}$ interval [highlighted in grey in Figures 3 (b) and (c)], experimental data still follow such linear behavior expected when non-interacting punctual heat sources within an infinite media are dissipating power. ${ }^{14,19}$ Thus, by employing behavioral modeling, increasing camera acquisition time, or even using a low pass filter, spatial noise in $\phi_{\tau}$ can be overcome. Secondly, this criterion should be different when heat sources 
interact, as deduced from Figure 3. In Figure 3 (b) around $M_{2}$ location, $\left.\phi_{\tau}\right|_{M_{2}}$ has another slope $\alpha_{D}{ }_{D}$ (in solid blue) whose inverse provides a new characteristic length $L^{\prime}{ }_{\mathrm{D}}=258 \mu \mathrm{m}$. This distance can be a new criterion to discriminate purely thermal interacting heat sources (i.e., substrate heat diffusion). From visual inspections in Figure 3 (a), the location of all IC parts can be deduced at shorter distances than those indicated above (approximately $40 \mu \mathrm{m}$ or less). According to this, the distances between $H S_{2}-M_{2}$ and $H S_{1}-M_{2}(270 \mu \mathrm{m}$, approximately) are long enough to detect a relative minimum in $\left.\phi_{\tau}\right|_{L_{\text {in }}}$ originated by a heat source caused by a substrate current.

As another interesting result, the real power averaged over $L_{\text {in }}$ and $M_{2}\left(|\bar{P}|_{L_{\text {in }}}\right.$ and $|\bar{P}|_{M_{2}}$, respectively) at $f=2 \mathrm{GHz}$ can be estimated gathering $\phi_{\tau}$ and $|\Delta T|$ results with electrical measurements of a virtual network analyzer (ZVM Rohde \& Schwarz). First, the output power $(35 \mu \mathrm{W})$ and reflected input power coefficient $(15 \%)$ at $f=2 \mathrm{GHz}$ are required. With these values, it can be calculated that $71 \%$ of $\mid P_{\text {in,het }} / 2$ (a half of $\left|P_{\text {in,het }}\right|$ per $f_{1}$ and $f_{2}$ ) is mainly dissipated into heat in $L_{\text {in }}$ and $M_{2}$ [according to Figures 2 (b) and (c)]. Next, from the thermal amplitude averaged in $L_{\text {in }}\left(|\overline{\Delta T}|_{L_{\text {in }}}\right)$ and $M_{2}\left(|\overline{\Delta T}|_{M_{2}}\right),|\bar{P}|_{L_{\text {in }}}$ and $|\bar{P}|_{M_{2}}\left(|\bar{P}|_{i}\right.$, where $i=L_{\text {in }}$ or $\left.M_{2}\right)$ can be extracted with the following expression:

$$
|\bar{P}|_{i}=\frac{|\overline{\Delta T}|_{i}}{|\overline{\Delta T}|_{L_{\text {in }}}+|\overline{\Delta T}|_{M_{2}}} 0.71\left|P_{\text {in,het }}\right| / 2
$$

where $|\bar{P}|_{i}=\kappa|\overline{\Delta T}|_{i}$ with the same $\kappa$ for each device is assumed in a first approximation. Thus, taking into account $|\overline{\Delta T}|_{L_{\text {in }}}$ and $|\overline{\Delta T}|_{M_{2}}$ at $f=2 \mathrm{GHz}$ and $\left|P_{\text {in,het }}\right| / 2$ (i.e., $\left.\left|P_{\text {in,het }}\right| / 2=250 \mu \mathrm{W}\right) ;|\bar{P}|_{L_{\text {in }}}$ and $|\bar{P}|_{M_{2}}$ are $76.3 \pm 15.4$ and $101.1 \pm 20.5 \mu \mathrm{W}$, respectively. Such values are not high enough to induce the amplifier failure, but in another case, the IC proper operation could be compromised. 
In summary, $\phi_{\tau}$ maps allow an on-line characterization of current paths and IC parts with a higher resolution than $|\Delta T|$ maps. $\phi_{\tau}$ can be used to improve the IC reliability and performances by design, or to locate the parts of the IC layout responsible for a yield drop. Another benefit when combined with $|\Delta T|$ measurements is not only the capability of inferring small-signal equivalent models by tracking current paths, but also to gain insight into the IC electrical behavior and quantitatively determine the components power consumption at any working frequency. This shows a great potential in future studies dealing with amplifier efficiency, induced overloading conditions in IC blocks, and other issues due to the connection tracks. Besides, this approach can be extended to other applications regardless of their working frequency, as for instance, the microstrip filters characterization.

This work has been partially supported by the Spanish Ministry of Science and Innovation (Research Programs TrenchSiC TEC2011-22607, SMARTCELLS TEC2014-51903-R, TEC2013-45638-C3-2-R, and Ramon y Cajal RYC-2010-07434) and by Generalitat de Catalunnya from AGAUR funds (2014-SGR 1596). 


\section{Figures \& Captions:}

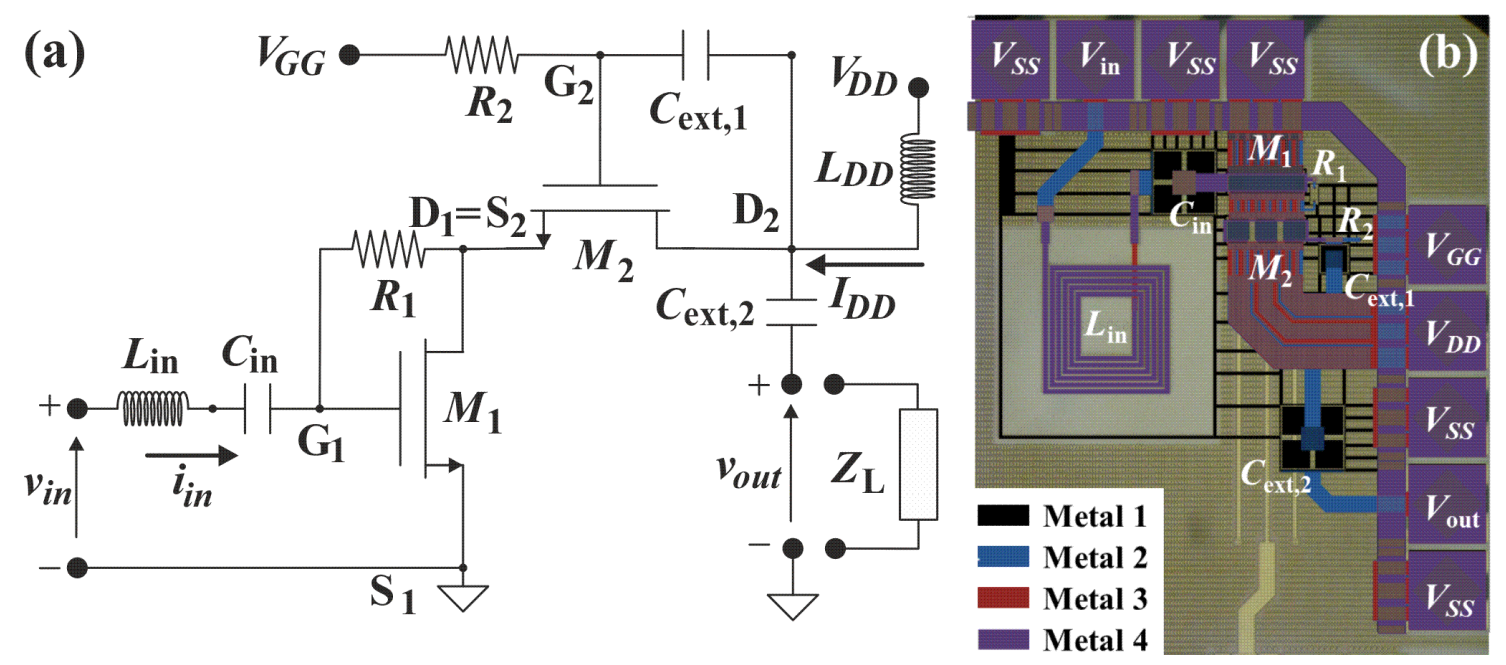

Figure 1. (a) Schematic of the cascode power amplifier analyzed. (b) Layout view of power amplifier, highlighting its in/output pads and pinout, main interconnection tracks (power supply, $V_{D D}$, Ground, $V_{\mathrm{SS}}$, input, $V_{\text {in }}$, and output, $\left.V_{\text {out }}\right)$, active $\left(M_{1}\right.$ and $\left.M_{2}\right)$ and passive ( $C_{\mathrm{in}}, L_{\mathrm{in}}, C_{\mathrm{ext}, 1} C_{\mathrm{ext}, 2}, R_{1}$ and $\left.R_{2}\right)$ components.

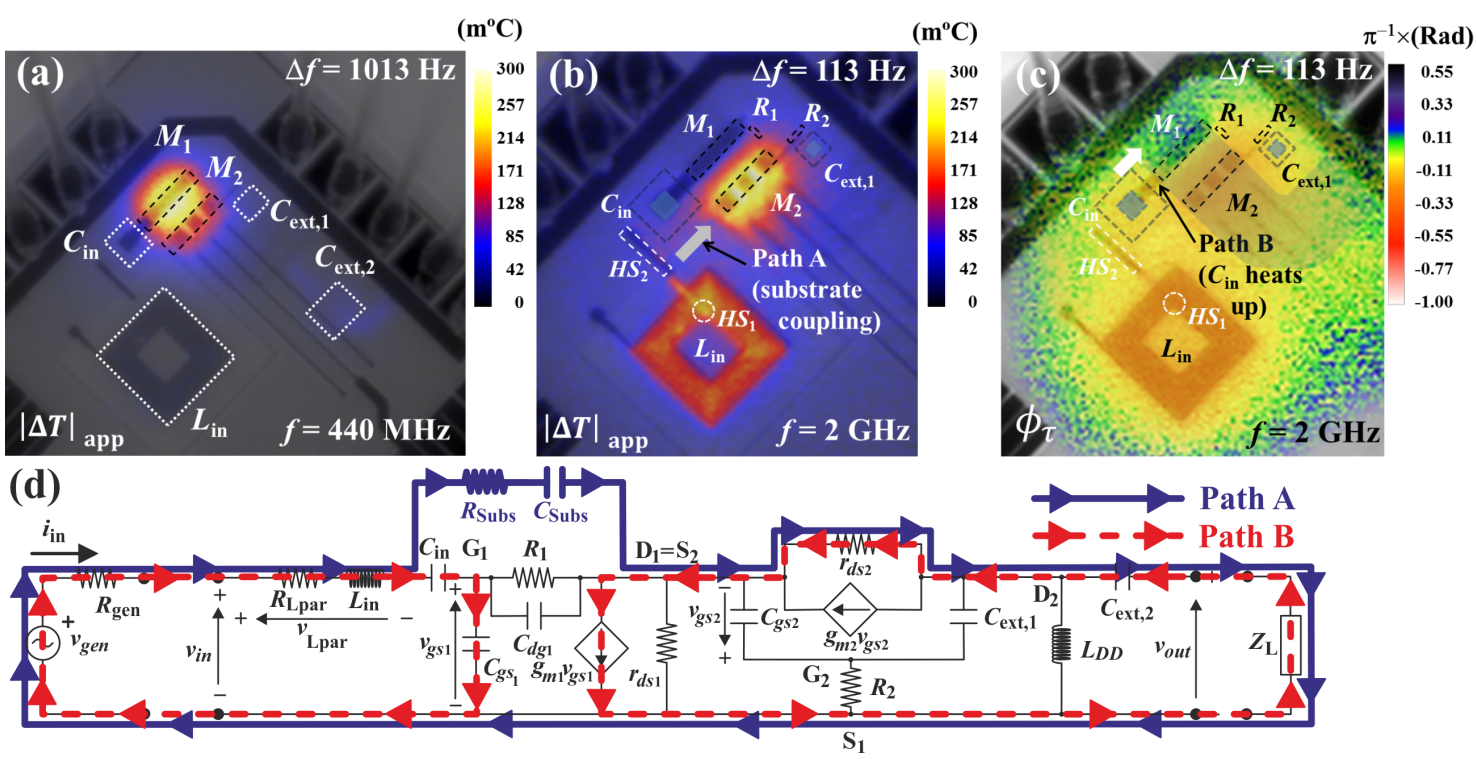

Figure 2. (a) $|\Delta T|_{\text {app }}$ map carried out at $\Delta f=1013 \mathrm{~Hz}$ and $f=440 \mathrm{MHz}$. (b) $|\Delta T|_{\text {app }}$ map performed at $\Delta f=113 \mathrm{~Hz}$ and $f=2 \mathrm{GHz}$. (c) $\phi_{\mathrm{r}}$ map performed at $\Delta f=113 \mathrm{~Hz}$ and $f=2 \mathrm{GHz}$. (d) Main paths taken by $i_{\text {in }}$ at $f=2 \mathrm{GHz}$ indicated in an amplifier's smallsignal equivalent circuit pointing out the path provided by substrate capacitive coupling or Path A (solid blue line), and that expected without coupling or Path B (dashed red line). 




Figure 3. $\phi_{\tau}$ map (at $f=2 \mathrm{GHz}$ and $\Delta f=113 \mathrm{~Hz}$ ) superposed to physical layout (a), correlating IC devices and components location with heat sources, and also indicating the direction of performed lateral profiles $\left.\phi_{\tau}\right|_{M_{2}}$ and $\left.\left.\phi_{\tau}\right|_{L_{\text {in }}} \cdot \phi_{\tau}\right|_{M_{2}}$ and $\left.\phi_{\tau}\right|_{L_{\text {in }}}$ profiles are presented in (b) and (c) respectively, pointing out the location of heat sources detected by $\phi_{\tau}$ measurements, IC area with noisy $\phi_{\tau}$ readings (highlighted in grey), and performed average (in black), jointly with the fitted linear expression with $x$ to extract the slopes $\alpha_{1}, \alpha_{2}$ (in red), and $\alpha_{\mathrm{D}}^{\prime}$ (in blue).

\section{References}

${ }^{1}$ A. M. Nikinejad, D. Chowdhury, and J. Chen, IEEE Trans. Microw. Theory Techn., 60 (2012).

2 J. J. Dabrowski, and R. M. Ramzan, IEEE Trans Very Large Scale Integr. (VLSI) Syst., 18, 933 (2010).

${ }^{3}$ J. Altet, D. Gomez, X. Perpinyà, D. Mateo, J. L. González, M. Vellvehi, and X. Jordà, Sensor. Actuat. A-Phys, 192, 49 (2013).

${ }^{4}$ P. J. Petersan, and S. M. Anlage, J. Appl. Phys., 84, 3392 (1998). 
${ }^{5}$ J. Altet, A. Rubio, J. L. Rossello, and J. Segura, IEEE Commun. Mag., 41, 98 (2003).

${ }^{6}$ S. R. Helmi, J. H. Chen, and S. Mohammadi, 2014 IEEE MTT-S International Microwave Symposium (IMS2014), Tampa, FL, 2014, pp. 1-3.

${ }^{7}$ J. H. Chen, S. R. Helmi, R. Azadegan, F. Aryanfar, and S. Mohammadi, IEEE J. Solid-State Circuits, 48, 2775 (2013).

${ }^{8}$ J. H. Chen, S. R. H., H. Pajouhi, Y. Sim, and S. Mohammadi, IEEE Trans. Microw. Theory Techn., 60, 4089 (2012).

${ }^{9}$ O. Breitenstein, W. Warta, and M. Langenkamp, Lock-in Thermography: Basics and Use for Evaluating Electronic Devices and Materials, (Springer Verlag, $2^{\text {nd }}$ edition, Berlin, 2010).

${ }^{10}$ M Farzaneh, K Maize, D Lüerßen, J A Summers, P M Mayer, P E Raad, K P Pipe, A Shakouri, R J Ram, and Janice A Hudgings, Phys. D: Appl. Phys. 42, 143001 (2009).

${ }^{11}$ A. Tosi, A. Dalla Mora, F. Pozzi, and F. Zappa, IEEE Electron. Device Lett., 29, 350(2008).

${ }^{12}$ J. Altet, W. Claeys, S. Dilhaire, A. Rubio, Proc. of the IEEE, 94, 1519 (2006).

${ }^{13}$ X. Perpinà, X. Jordà, N. Mestres, M. Vellvehi, P. Godignon, J. Millán, and H. von Kiedrowski, Meas. Sci. Tech., 15, 1011 (2004).

${ }^{14}$ X. Perpiñà, X Jordà, M Vellvehi, and J Altet, Appl. Phys. Lett., 105, 084101 (2014).

${ }^{15}$ S. Y. Suck, G. Tessier, N. Warnasooriya, A. Babuty, and Y. De Wilde, Appl. Phys. Lett., 96, 121108 (2010).

${ }^{16}$ L. Aigouy, G. Tessier, M. Mortier, and B. Charlot, Appl. Phys. Lett., 87, 184105 (2005).

17 J. León, X. Perpiñà, M. Vellvehi, A. Baldi, J. Sacristán, and X. Jordà, Appl. Phys. Lett., 102, 084106 (2013).

${ }^{18}$ J. León, M. Berthou, X. Perpiñà, J. Montserrat, M. Vellvehi, P. Godignon, and X. Jordà, J. Phys. D, 47, 055102 (2014).

${ }^{19}$ J. León, X. Perpiñà, J. Altet, M. Vellvehi, and X. Jordà, Appl. Phys. Lett., 102, 054103 (2013).

${ }^{20}$ A. Shakouri, Proc. of IEEE, 94, 1613 (2006).

${ }^{21}$ M. Montes Bajo, C. Hodges, M. J. Uren, and M. Kuball, Appl. Phys. Lett., 101, 033508 (2012).

${ }^{22}$ R. Ouaida, M. Berthou, J. León, X. Perpiñà, S. Oge, P. Brosselard, and C. Joubert, IEEE Electron Device Lett., 35, 1284 (2014).

${ }^{23}$ F. Fertig, J. Greulich, and S. Rein, Appl. Phys. Lett., 104, 201111 (2014).

${ }^{24}$ F. Frühauf, Y. Sayad, and O. Breitenstein, Sol. Energ. Mat. Sol. C., 154, 23 (2016).

${ }^{25}$ R. C. Redondo, N. R. Melchor, F. R. Quintela, and M. Redondo, Int. J. Elec. Power, 45, 369 (2015).

${ }^{26}$ X. Perpiñà, J. Altet, X. Jordà, M. Vellvehi, and N. Mestres, Opt. Lett., 35, 2657 (2010)

${ }^{27}$ Y. Cao, V. Issakov, and M. Tiebout, Proc. of $4^{\text {th }}$ IEEE Solid-State Circuits Conference, San Francisco (USA), pp. 194-606, 2008

${ }^{28}$ P. E. Allen, and D. R. Holberg, CMOS Analog Circuit Design, (Oxford University Press, 2nd edition, New York, 2002), pp. 199-211.

${ }^{29}$ X. Perpiñà, X. Jordà, M. Vellvehi, J. Altet, and N. Mestres, J. Phys. D, 41, 155508 (2008). 